\title{
Anew species of Myxia (Hemiptera: Auchenorrhyncha: Cixiidae) collected on palms from the Reserva Privada el Silencio de Los Angeles Cloud Forest in Costa Rica
}

\author{
MARCO A. ZUMBADO ECHAVARRIA ${ }^{1}$, EDWIN A. BARRANTES BARRANTES ${ }^{2}$, CHARLES R. BARTLETT $^{3}$, \\ ERICKA E. HELMICK ${ }^{4} \&$ BRIAN W. BAHDER ${ }^{* *}$ \\ ${ }^{1}$ Universidad de Costa Rica-Sede San Ramón, Departamento de Ciencias Naturales, de la Iglesia el Tremedal 400 mts al Oeste car- \\ retera hacia San Pedro, San Ramón, Alajuela, Costa Rica. \\ "=marco.zumbado@ucr.ac.cr; nassua75@gmail.com; ○ https://orcid.org/0000-0002-2591-7662 \\ ${ }^{2}$ Universidad de Costa Rica-Sede San Ramón, Departamento de Ciencias Naturales, de la Iglesia el Tremedal 400 mts al Oeste car- \\ retera hacia San Pedro, San Ramón, Alajuela, Costa Rica. \\ "edwin.barrantes@ucr.ac.cr; edwbarrantes@gmail.com; @ https://orcid.org/0000-0001-9565-2105 \\ ${ }^{3}$ University of Delaware, Department of Entomology and Wildife Ecology, 250 Townsend Hall, Newark, DE 19716-2160, USA. \\ "'bartlett@udel.edu; @ https://orcid.org/0000-0001-9428-7337 \\ ${ }^{4}$ University of Florida, Department of Entomology and Nematology_Fort Lauderdale Research and Education Center; 3205 College \\ Ave., Davie, FL 33314-7719, USA. \\ ”"ehelmick@ufl.edu; @ ittps://orcid.org/0000-0002-5153-0891 \\ ${ }^{5}$ University of Florida, Department of Entomology and Nematology_Fort Lauderdale Research and Education Center; 3205 College \\ Ave., Davie, FL 33314-7719, USA. \\ "'bbahder@ufl.edu; @ https://orcid.org/0000-0002-1118-4832 \\ *Corresponding author. !" bbahder@ufl.edu
}

\begin{abstract}
A new species of Myxia Bahder \& Bartlett (Cixiidae: Cixiinae: Oecleini) is established as Myxia hernandezi sp. n. collected from native palms in cloud forest habitat in Costa Rica. Placement in the genus Myxia is supported by molecular analysis of the cytochrome $c$ oxidase subunit I (COI) and 18S loci as well as morphological characters.
\end{abstract}

Key words: Cixiidae, taxonomy, planthopper, palm, Costa Rica

\section{Resumen}

Una nueva especie del género Myxia Bahder \& Bartlett (Cixiidae: Cixiinae: Oecleini) fue encontrada en palmas nativas en el hábitat de bosque nuboso en Costa Rica. Esta nueva especie se denomina Myxia hernandezi sp. n. Su colocación bajo el género Myxia está respaldada por el análisis molecular de la subunidad I del citocromo $c$ oxidasa (COI) y los loci 18S, así como por sus caracteres morfológicos.

Palabras clave: Cixiidae, taxonomía, chicharrita, palmera, Costa Rica

\section{Introduction}

Myxia Bahder \& Bartlett, 2019 was recently established to accommodate Myxia belinda Bahder \& Bartlett, 2019 (in Bahder et al. 2019a) discovered on palms in Costa Rica. An additional Myxia species was subsequently described from the same region, also on palms and M. delta (Kramer, 1979) was transferred from Haplaxius Fowler, 1904 based on molecular and morphological support (Echavarria et al. 2021). The genus Myxia now consists of three species with a Mesoamerican distribution. All Myxia species were documented while surveying planthoppers on palms pursuant to a renewed interest in the host/vector relationships between cixiids and palm phytoplasmas. Haplaxius crudus (Van Duzee, 1907) has long been asserted to be involved in the transmission of lethal yellowing (LY) phy- 
toplasmas (e.g., Howard \& Thomas 1980) and more recently evidence as its role as a vector for other subgroups of phytoplasmas in the palm lethal decline group (16SrIV) (Dzido et al. 2020).

The genus Myxia is placed in the tribe Oecleini (Cixiinae) and allied with Haplaxius and Myndus Stål, 1862, but distinct based on molecular and morphological evidence. Historically, Haplaxius and Myndus had been synonyms (e.g., Kramer 1979) but Haplaxius was reinstated as valid by Emeljanov (1989). Functionally Haplaxius is New World and Myndus Old World, diagnosed by the absence (Haplaxius) or presence (Myndus) of a denticle on the forecoxae and carinae terminating on the ventro-lateral corner of the prothorax (Haplaxius) or approximately midway (Myndus). In species of Myxia, the denticles of the forecoxae are lacking. The pronotal carinae appeared to terminate midway on the ventral margins of Myxia, similar to Myndus; however, M. delta has the carinae terminating as in Haplaxius. Based on molecular data, it appears the termination of pronotal carinae is not a reliable character for recognizing Myxia.

The terminalia in Myxia are distinctive. Compared with species of Haplaxius considered by Kramer (1979), the presence of a subtriangular medioventral process of the pygofer, distally bilobed gonostyli, and simple aedeagus subtended by a complex periandrium ( $\approx$ phallobase) appear to be synapomorphies of Myxia.

Herein, we describe a novel species in Myxia collected from palms in the genus Geonoma Willd. We obtained the cytochrome $c$ oxidase subunit I (COI), 18S rRNA, and histone 3 (H3) genes of the new species for phylogenetic analyses with other members of the tribe Oecleini.

\section{Materials and methods}

Locality and specimen collection. Individuals of the novel taxon were collected from a palm (Arecaceae) belonging to the genus Geonoma Willd., 1805 in the Reserva Privada el Silencio de Los Angeles Cloud Forest in Costa Rica at the Hotel Villa Blanca, Alajeula Province (10.203231, -84.485094). Specimens were aspirated directly from the palms and transferred to $95 \%$ ethanol in the field while still alive. After a few minutes of disturbance, individuals became more active and were subsequently collected by sweep net. Specimens were collected (permit no. SINACACTo-GASPPNI-016-2018) with permission of the Hotel Villa Blanca management and staff and exported under permit number DGVS-256-2018 to the U.S.A. and imported under permit number P526-170201-001. Additionally, a specimen identified in the field as a Haplaxius species was collected from a coconut palm in Manzanilla, along the Caribbean coast near the Panamanian border. All specimens collected were measured, photographed and dissected using a Leica M205 C stereoscope. Images of specimens and all features photographed were generated using the LAS Core Software v4.12. Voucher specimens, including primary types, are stored at the University of FloridaFort Lauderdale Research and Education Center (FLREC) in Davie, FL, U.S.A and the Florida State Collection of Arthropods (FSCA) in Gainesville, FL, U.S.A.

Morphological terminology and identification. Morphological terminology generally follows Kramer (1979) except with male terminalia nomenclature updated after Bourgoin (1988) and Bourgoin \& Huang (1990) and forewing venation following Bourgoin et al. (2015). New taxa are to be attributed to Bahder and Bartlett.

Dissections and DNA extraction. The terminalia that were dissected also served as the source of tissue for DNA extraction. The terminal end of the abdomens was removed and placed directly into a solution of tissue lysis buffer (buffer ATL) and proteinase K (180 $\mu$ ATL and $20 \mu$ l proteinase K) from the DNeasy ${ }^{\circledR}$ Blood and Tissue Kit (Qiagen). The abdomen was left to lyse for 24 hours at $56^{\circ} \mathrm{C}$. Following lysis, eluate was transferred to a new 1.5 $\mathrm{ml}$ microcentrifuge tube and DNA extraction proceeded as per the manufacturer's instructions. The terminalia were then immersed in $200 \mu \mathrm{l}$ of buffer ATL and $200 \mu \mathrm{l}$ of buffer AL from the same kit and placed at $95^{\circ} \mathrm{C}$ for 24 hours to remove fat, wax, and residual tissue. The cleared genitalia were then used for morphological characterization and photography.

PCR parameters, sequence data, and analysis. To obtain COI for the novel taxon a degenerate forward primer was designed using available COI data from cixiids in the tribe Oecleini in GenBank due to failed attempts to amplify using primers in Echavarria et al. (2021) and the reverse primer was the reverse compliment of the C1J-2195 primer by Simon et al. (1994). To obtain $18 \mathrm{~S}$ sequence data, the primers developed by Bahder et al. (2019b) were used and to obtain H3 sequence data, sequence available from Urban \& Cryan 2007 were aligned and degenerate primers were designed visually. All primers used in this study are presented in Table 1. PCR reactions contained 5x GoTaq Flexi Buffer, $25 \mathrm{mM} \mathrm{MgCl}, 10 \mathrm{mM}$ dNTP's, $10 \mathrm{mM}$ of each primer, 10\% PVP-40, and 2.5U GoTaq Flexi 
DNA Polymerase, $2 \mu 1$ DNA template, and sterile $\mathrm{dH}_{2} 0$ to a final volume of $25 \mu \mathrm{L}$. Thermal cycling conditions for all loci involved were as follows: 2 min initial denaturation at $95^{\circ} \mathrm{C}$, followed by 35 cycles of $30 \mathrm{sec}$ denaturation at $95^{\circ} \mathrm{C}, 30 \mathrm{sec}$ annealing, and extension at $72^{\circ} \mathrm{C}$. Specific annealing temperatures and extension times for respective loci are presented in Table 1. Products were visualized on a 1.5\% agarose gel stained with GelRed (Biotium). PCR products of the appropriate size were purified using the ExoSAP-ITTM Express PCR Product Cleanup Reagent per the manufacturers' protocol (ThermoFisher Scientific, Waltham, Massachusetts, USA). Purified PCR product was quantified using a NanoDrop Lite Spectrophotometer (ThermoFisher Scientific, Waltham, Massachusetts, USA) and sequenced using the SeqStudio Genetic Analyzer (Applied Biosystems). Contiguous files were assembled using DNA Baser (Version 4.36) (Heracle BioSoft SRL, Pitesti, Romania), aligned using ClustalW as part of the package MEGA7 (Kumar et al. 2016). A matrix of pairwise differences using number of differences among COI, 18S, and H3 was calculated with MEGA7 (Kumar et al. 2016). The bootstrap method was used for variance estimation at 1,000 replicates and using the p-distance model. Maximum Likelihood trees were generated using the Bootstrap method at 1,000 replicates based on the Tamura-Nei model for both the COI, 18S, and H3 loci as well as the consensus tree with concatenated data for COI and $18 \mathrm{~S}$ data.

TABLE 1. Primers used to obtain molecular data used to support placement of novel taxon and critical parameters for PCR success with respective primers.

\begin{tabular}{lllllc}
\hline Locus & Primer & Direction & Sequence $\left(5^{\prime} \rightarrow 3^{\prime}\right)$ & Annealing & Extension \\
\hline COI & COI_D1_F & Forward & GGAACWATAAGAAGWATAATYATYCG & $40^{\circ} \mathrm{C}$ & 1 min. $30 \mathrm{sec}$. \\
& C1-J-2195RC & Reverse & ACTTCTGGATGACCAAAAAATCAA & & \\
$18 \mathrm{~S}$ & $18 \mathrm{SF}$ & Forward & ACTGTCGATGGTAGGTTCTG & $50^{\circ} \mathrm{C}$ & $2 \mathrm{~min}$. \\
& $18 \mathrm{SR}$ & Reverse & GTCCGAAGACCTCACTAAA & & \\
$\mathrm{H} 3$ & $\mathrm{H} 3 \mathrm{~F}$ & Forward & CAGACGGCBMGKAARTCSACC & $55^{\circ} \mathrm{C}$ & $30 \mathrm{sec}$. \\
& $\mathrm{H} 3 \mathrm{R}$ & Reverse & GTKACHCKCTTRGCGTGRAT & & \\
\hline
\end{tabular}

Taxon sampling. For molecular comparisons M. baynardi Bahder \& Bartlett, 2021, M. belinda and M. delta were used for in-group comparison and out-groups included five species of Haplaxius - H. crudus, H. dougwalshi Bahder \& Bartlett, 2020 (in Bahder et al., 2020), H. pictifrons (Stål, 1862), H. pocococo Bahder \& Bartlett 2021 (in Barrantes et al. 2021) and H. skarphion (Kramer, 1979), one species of Oecleus - O. mackaspringi Bahder \& Bartlett, 2019 (in Myrie et al. 2019) and Nymphomyndus caribbea (Fennah, 1971). All in-group and out-group taxa data, including GenBank accession numbers are presented in Table 2.

TABLE 2. Taxon sampling of Oecleini for molecular study.

\begin{tabular}{llccll}
\hline & & \multicolumn{3}{c}{ GenBank Accession No. } \\
\cline { 3 - 5 } Taxon & Locality & COI & 18S & H3 & Collection \\
\hline Haplaxius crudus & Costa Rica & MT080284 & MT002393 & MZ274037 & FLREC \\
Haplaxius dougwalshi & Costa Rica & MT080284 & MT002395 & MZ297815 & FLREC \\
Haplaxius pictifrons & Delaware & MT946292 & MN200098 & MZ274038 & FLREC \\
Haplaxius skarphion & Costa Rica & MT900603 & MT892907 & MZ274039 & FLREC \\
Myxia baynardi & Costa Rica & MT900604 & MT892909 & MZ274040 & FLREC \\
Myxia belinda & Costa Rica & MT900605 & MN200095 & MZ274041 & FLREC \\
Myxia delta & Costa Rica & MT900602 & MT892907 & MZ274042 & FLREC \\
Nymphomyndus caribbea & Jamaica & MT080286 & MT002394 & MZ274044 & FLREC \\
Oecleus mackaspringi & Jamaica & MN488999 & MN422261 & MZ274045 & FLREC \\
\hline
\end{tabular}

\section{Results}

\section{Systematics}

Family Cixiidae Spinola, 1839 
Subfamily Cixiinae Spinola, 1839

\section{Tribe Oecleini Muir, 1922}

\section{Genus Myxia Bahder \& Bartlett, 2019}

Type species: Myxia belinda Bahder \& Bartlett, 2019; by monotypy and original designation.

Diagnosis. Vertex longer at midline than wide, median carina present, evanescent distally. Hind tibiae lacking lateral spines (a tribal feature), forecoxae lacking denticle. Medioventral process of pygofer subtriangular. Parameres distally bifid in lateral view, with acute apex in ventral view. Periandrium ( $\approx$ phallobase) usually ring-like at base of aedeagus, prolonged into a projection of varying form subtending aedeagus. Anal tube short and stout, distally projecting ventrally or ventrocaudally.

\section{Key to the species of Myxia (males)}

1. Body dark fuscous, vertex bicolored (tannish caudally, fuscous distally), wings patterned, lacking ir crossvein (im also missing); flagellum large bearing four long sclerotized processes . . . . . . . . . . . . Myxia baynardi Bahder \& Bartlett, 2021

1'. Body whitish or yellow, vertex pale, not bicolored; wings clear, $i r$ crossvein present $\ldots \ldots \ldots \ldots \ldots \ldots \ldots \ldots \ldots \ldots \ldots \ldots \ldots$

2. Body uniformly yellow, face without markings, periandrium with large retrorse spine and two subapical anterior angled spines . . . . . . . . . . . . . . . . . . . . . . . . . . . . . . . . . Myxia belinda Bahder \& Bartlett, 2019

2'. Body whitish, face with white markings and orange patch traversing median carina $\ldots \ldots \ldots \ldots \ldots \ldots \ldots$

3. Aedeagal shaft in ventral view with one fin-shaped process on right lateral side (Bahder et al. 2019a, fig. 8C), a single process at endosomal apex, medioventral process of pygofer more rounded (Bahder et al. 2019a, fig. 7B) . . . . . . . . . . . . . .

............................................................. Myxia delta (Kramer, 1979)

3'. Aedeagal shaft with two fin-shaped processes, one on each side (Figs. 5B, 6C), two processes at endosomal apex (Fig. 6D),

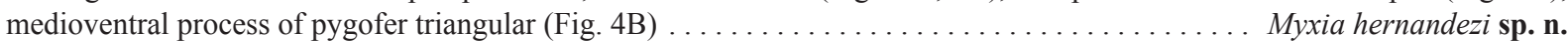

\section{Myxia hernandezi Bahder \& Bartlett sp. n.}

(Figures 1-6)

Type locality. Costa Rica, Alajuela, Reserva Privada el Silencio de Los Angeles, Hotel Villa Blanca.

Diagnosis. Body yellowish, frons with "M-shaped" white marking and median light orange patch traversing median carina. Vertex longer at midline than wide at base, median carina present, evanescent distally. Hind tibiae lacking spines, forecoxae lacking denticle at distal end of trailing margin. Medioventral process of pygofer opening large, subtriangular. Gonostyli distally bifid in lateral view, dorsal process hooked cephalad, in ventral view with tooth in distal third of medial margin. Periandrium subtending and broadly fused to aedeagus, in lateral view (Fig. 5B) apically prolonged into two robust, ventrally hooked, apical spines. Aedeagal shaft with two lateral, fin-like processes curved ventrad, endosoma membranous, curved dextrally, bearing two subapical processes.

Description. Color. General body color yellow to yellowish-white, slightly darker on mid-dorsal tergites of abdomen, paler ventrally (Fig. 1). Legs near white. Head with frons bicolored, dorsally orangish and with elongate orangish patch on midline extending approximately $1 / 3$ length from frontoclypeal suture, outlining a white "Mshaped" patch on ventral half of frons, traversing entire width, (Fig. 2A). Lateral ocelli reddish. Forewings transparent, veins pale.

Structure. Body length (with wings), male $5.95 \mathrm{~mm}(\mathrm{n}=2)$, female $6.19 \mathrm{~mm}(\mathrm{n}=5)$; body length (without wings), male $4.42 \mathrm{~mm}$, female 4.51 (Table 3). Head. Head in dorsal view (including eyes) narrower than pronotum, in lateral view obtusely rounded from posterior margin of vertex to frontoclypeal suture (Fig. 2). Vertex from dorsal view roughly quadrate, disc concave, longer at midlength than broad at widest point (at posterior margin), lateral margins diverging posteriorly, anterior margin truncate at transverse carina, posterior margin medially incised with sinuate margins median carina nearly obsolete (most evident posteriorly) (Fig. 2). Frons broadly ovate, much longer than wide, sides convexly arched, narrowest between eyes, expanding ventrally to below antennae, narrowed at frontoclypeal suture, median carina evident, median ocellus distinct (Fig. 2A). Lateral ocelli conspicuous on genae 
below anterior margin of eyes (above level of antennae). Frontoclypeal suture straight, clypeus triangular (roughly equilateral), median carina evident. Antennae with scape very short, pedicel bulbous, about as wide as tall bearing conspicuous sensory plaques, flagellum elongate and bristle-like with bulbous base.

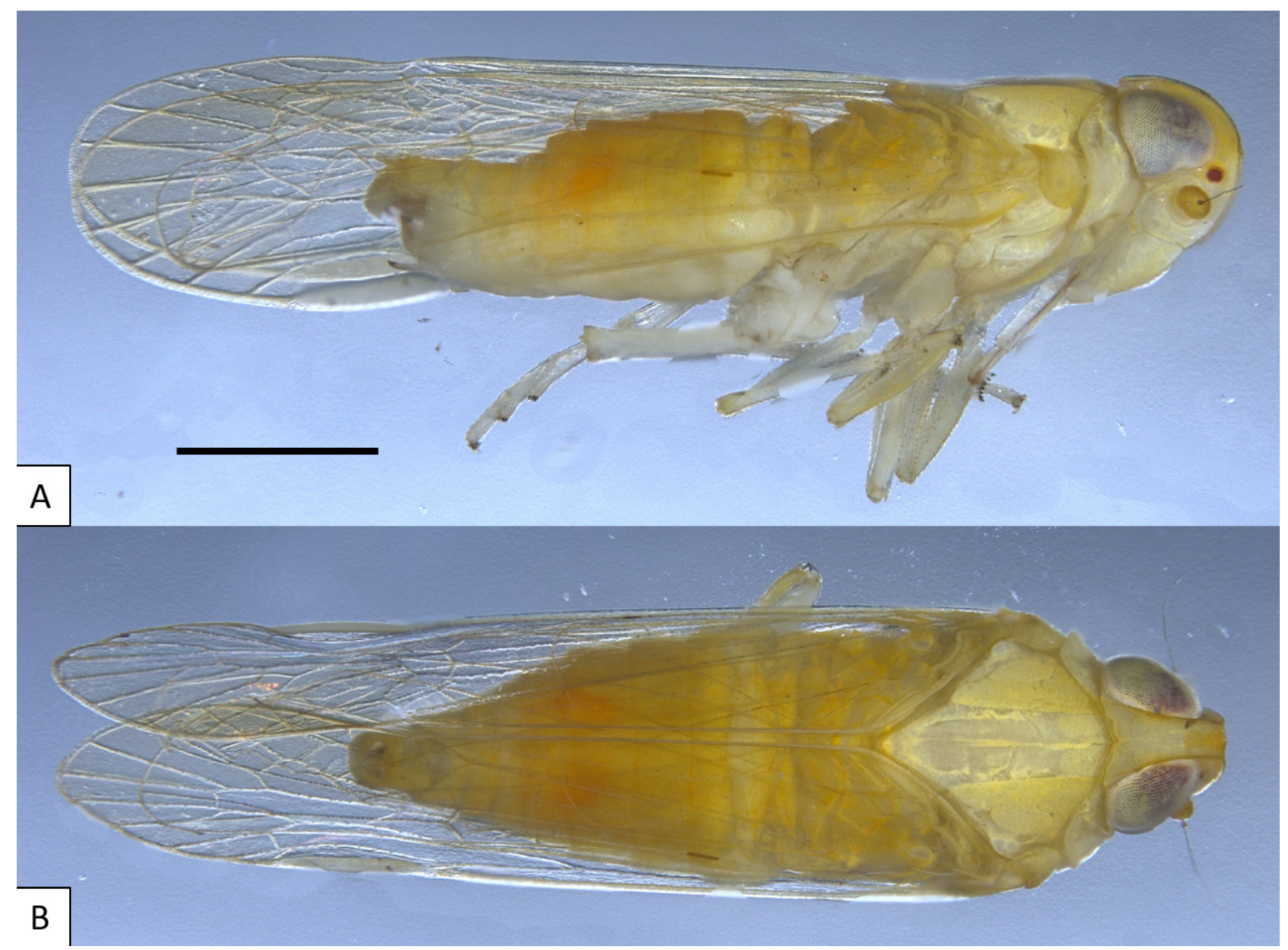

FIGURE 1. Adult male habitus Myxia hernandezi sp. n.; (A) body lateral view and (B) body dorsal view; scale = $1 \mathrm{~mm}$.

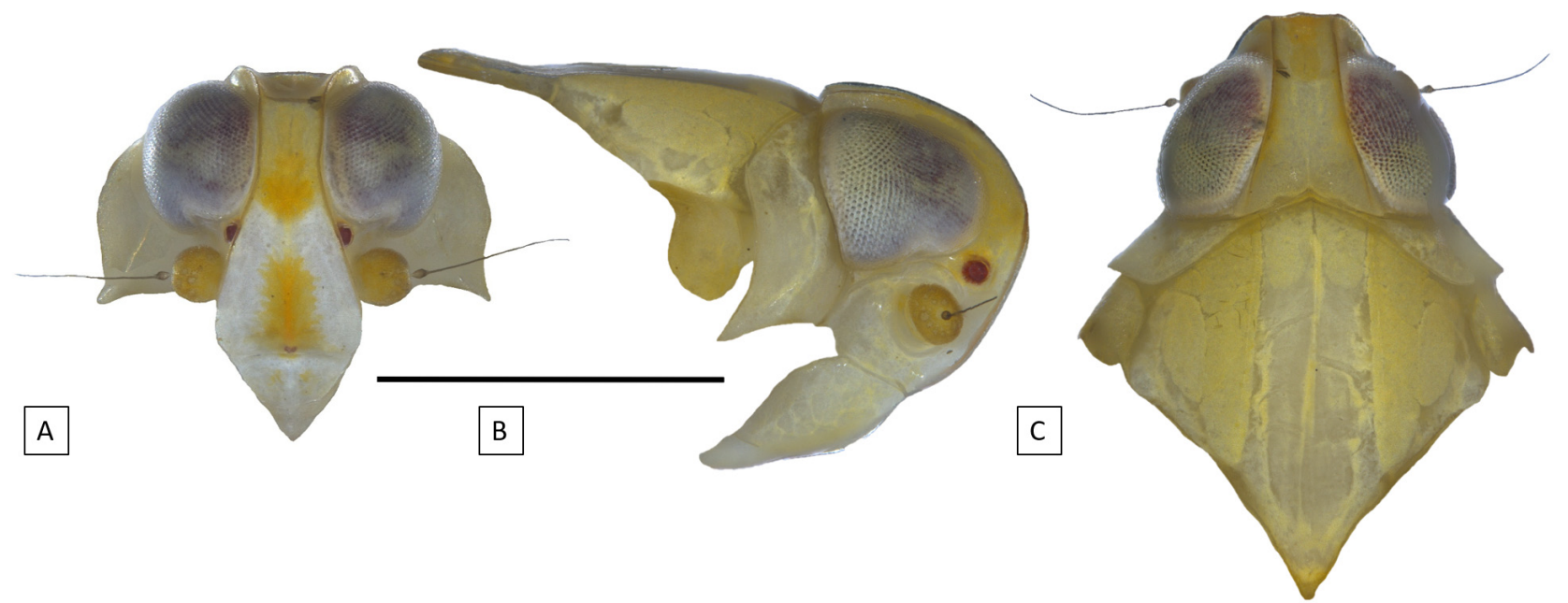

FIGURE 2. Figure 2. Adult Myxia hernandezi sp. n.; (A) male head and pronotum frontal view, (B) male head, pronotum and mesonotum lateral view, and (C) male head pronotum, and mesonotum dorsal view; scale $=1 \mathrm{~mm}$. 
TABLE 3. Biometric data of Myxia hernandezi sp. n. (measurements in millimeters [mm]).

\begin{tabular}{|c|c|c|c|c|}
\hline \multirow[b]{2}{*}{ Character } & \multicolumn{2}{|c|}{ Male $(n=2)$} & \multicolumn{2}{|c|}{ Female $(n=5)$} \\
\hline & Average \pm SE & Range & Average $\pm \mathrm{SE}$ & Range \\
\hline Body length with wings & $5.95 \pm 0.00$ & 5.95 & $6.19 \pm 0.00$ & 6.19 \\
\hline Body length without wings & $4.42 \pm 0.00$ & 4.41 & $4.51 \pm 0.00$ & 4.51 \\
\hline Forewing length & $4.97 \pm 0.00$ & 4.97 & $5.28 \pm 0.00$ & 5.28 \\
\hline Vertex length & $0.44 \pm 0.00$ & 0.34 & $0.36 \pm 0.00$ & 0.36 \\
\hline Vertex width—-basal & $0.34 \pm 0.00$ & 0.44 & $0.46 \pm 0.00$ & 0.46 \\
\hline Vertex width-distal & $0.21 \pm 0.00$ & 0.21 & $0.23 \pm 0.00$ & 0.23 \\
\hline Pronotum length — midline & $0.03 \pm 0.00$ & 0.03 & $0.03 \pm 0.00$ & 0.03 \\
\hline Mesonotum length — midline & $1.22 \pm 0.00$ & 1.22 & $1.27 \pm 0.00$ & 1.27 \\
\hline Mesonotum width & $1.12 \pm 0.00$ & 1.12 & $1.14 \pm 0.00$ & 1.14 \\
\hline Frons length & $0.78 \pm 0.10$ & 0.78 & $0.81 \pm 0.00$ & 0.81 \\
\hline Frons width—dorsal & $0.20 \pm 0.00$ & 0.20 & $0.22 \pm 0.00$ & 0.22 \\
\hline Frons width—frontoclypeal & $0.37 \pm 0.00$ & 0.37 & $0.39 \pm 0.00$ & 0.39 \\
\hline Frons width—widest & $0.46 \pm 0.00$ & 0.46 & $0.47 \pm 0.00$ & 0.47 \\
\hline Frons width-narrowest & $0.19 \pm 0.00$ & 0.19 & $0.20 \pm 0.00$ & 0.20 \\
\hline Clypeus length & $0.67 \pm 0.00$ & 0.67 & $0.69 \pm 0.00$ & 0.69 \\
\hline
\end{tabular}

Thorax. Pronotum in dorsal view very short (medially nearly hidden below posterior margin of vertex), concave posteriorly; median carina weak, lateral portions expanded, reaching tegulae, postocular carinae extending from pronotal midline to ventro-lateral corner of pronotum (Fig. 2C); in lateral view paradiscal region broad, extended to ventral level of antennae, posterior margin concave, forming point at ventroposterior margin. Mesonotum longer than wide; anterior margin roundly convex, tricarinate, lateral carinae nearly parallel, diverging slightly posteriorly, median carina weaker on scutellum (Fig. 2C); in lateral view mesonotum weakly concave at leading margin of scutellum (Fig. 2B). Spinulation for hind tarsal segments 8-8-7.

Fore wing. Fore wings elongate, costal and trailing margin subparallel (slightly expanded distally, weakly concave at apex of clavus); apex of clavus just past forewing midlength, Pcu+A1 fused about midlength of clavus (at about $1 / 3$ forewing length), reaching wing margin well before $\mathrm{CuP}$, fork of ScP+RA from RP near wing midlength, $\mathrm{CuA}$ fork nearly at claval margin distad of $\mathrm{ScP}+\mathrm{RA}$ from $\mathrm{RP}(\mathrm{C} 1$ cell much longer than $\mathrm{C} 5)$, $\mathrm{ScP}+\mathrm{R}+\mathrm{MP}$ fused at base forming long stem from basal cell. Branching pattern RA 2-branched (leading branch weak), RP 3-branched, MP 4-branched; CuA 2-branched (distally fused forming closed 'procubital cell' [i.e., Emeljanov 1996] and marginal C6 cell); crossveins ir and im present.

Terminalia. Pygofer in lateral view bell-shaped, narrowest dorsally, widest ventrally (Fig. 4), leading margin diagonal and weakly concave, trailing margin sinuate, weakly convex. In ventral view, medioventral process of pygofer opening subtriangular, large, longer than wide (midlength approximately $2 \times$ width at base) (Fig. 4B). Gonostyli in lateral view irregularly sinuate on both dorsal and ventral margin, widest near midlength, ventral margin with distinct lobe at base; subapical process on dorsal margin present, hooked cephalad (Fig. 4A); in ventral view, margins nearly parallel, narrowed and medially curved distally, sclerotized tooth on inner margin at $2 / 3$ length, apex diagonally truncate (Fig. 4). Periandrium fused to ventral side of aedeagus, asymmetrical, serrate on ventral margin (Fig. $5 \&$ 6), with distal lobe on dorsal margin (Fig. 6A, P1), two curved ventro-apical processes (P2 \& P3), and ventral a process on right lateral side, directed caudad (Fig. 6D, P4). Aedeagal shaft with two, lateral fin-like flaps near shaft midlength, left flap (Fig. 6A, A1) more robust than right (A2). Endosoma well developed, dextrally curved from aedeagal apex, with two sclerotized processes on ventral margin near apex (Fig. 6D), longer process on lateral margin (F1) and shorter process mesad (F2). Anal tube short and stout in lateral view, apex downcurved, weakly asymmetrical; ventral margin nearly straight to level of paraproct, more strongly concave distally (Fig. 4A); in dorsal view apex rounded; paraproct distinct, approximately columnar.

Plant associations. Palm (Geonoma sp.), Arecaceae.

Distribution. Costa Rica (Alajeula). 


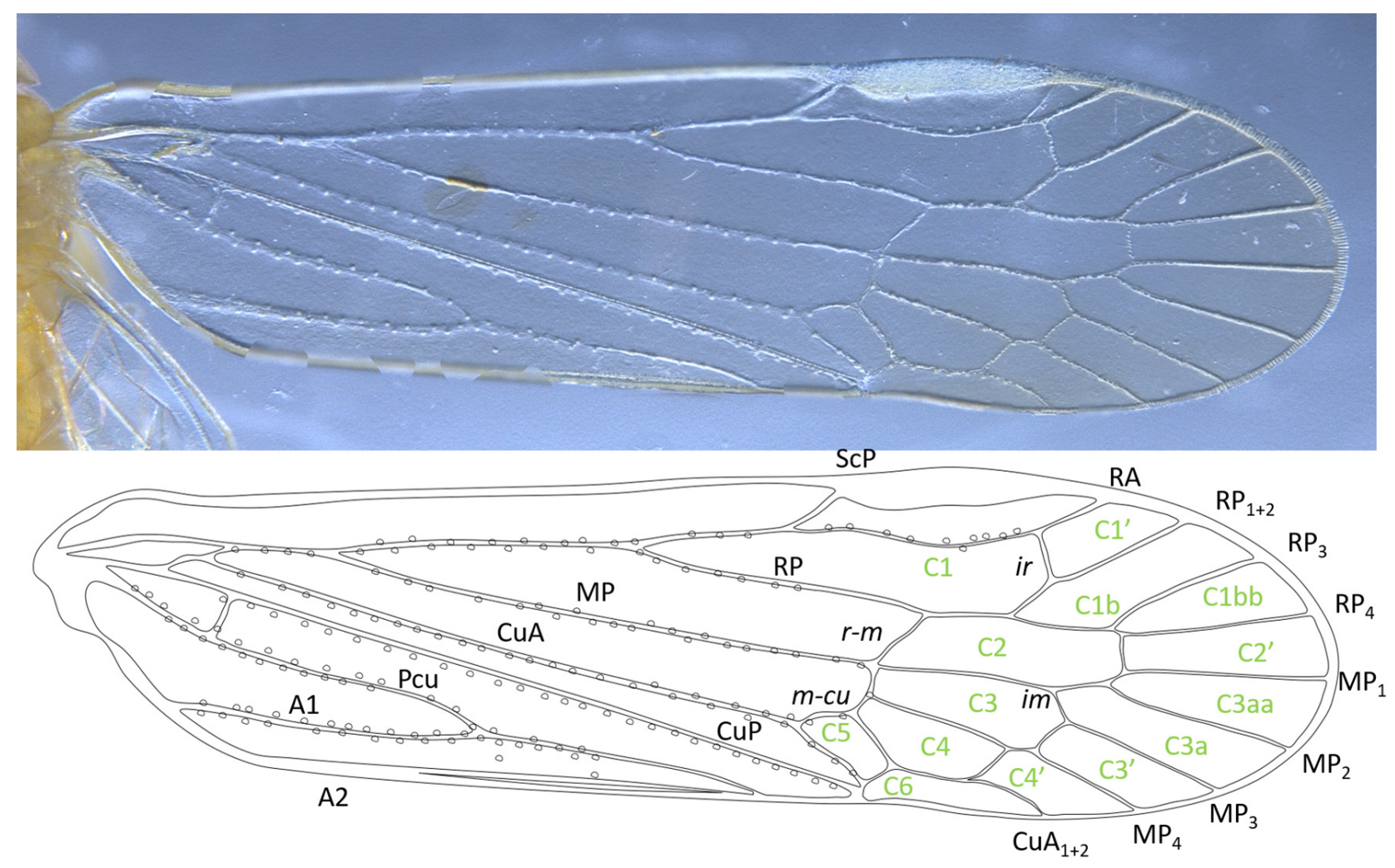

FIGURE 3. Forewing venation of Myxia hernandezi $\mathbf{s p .} \mathbf{n}$.
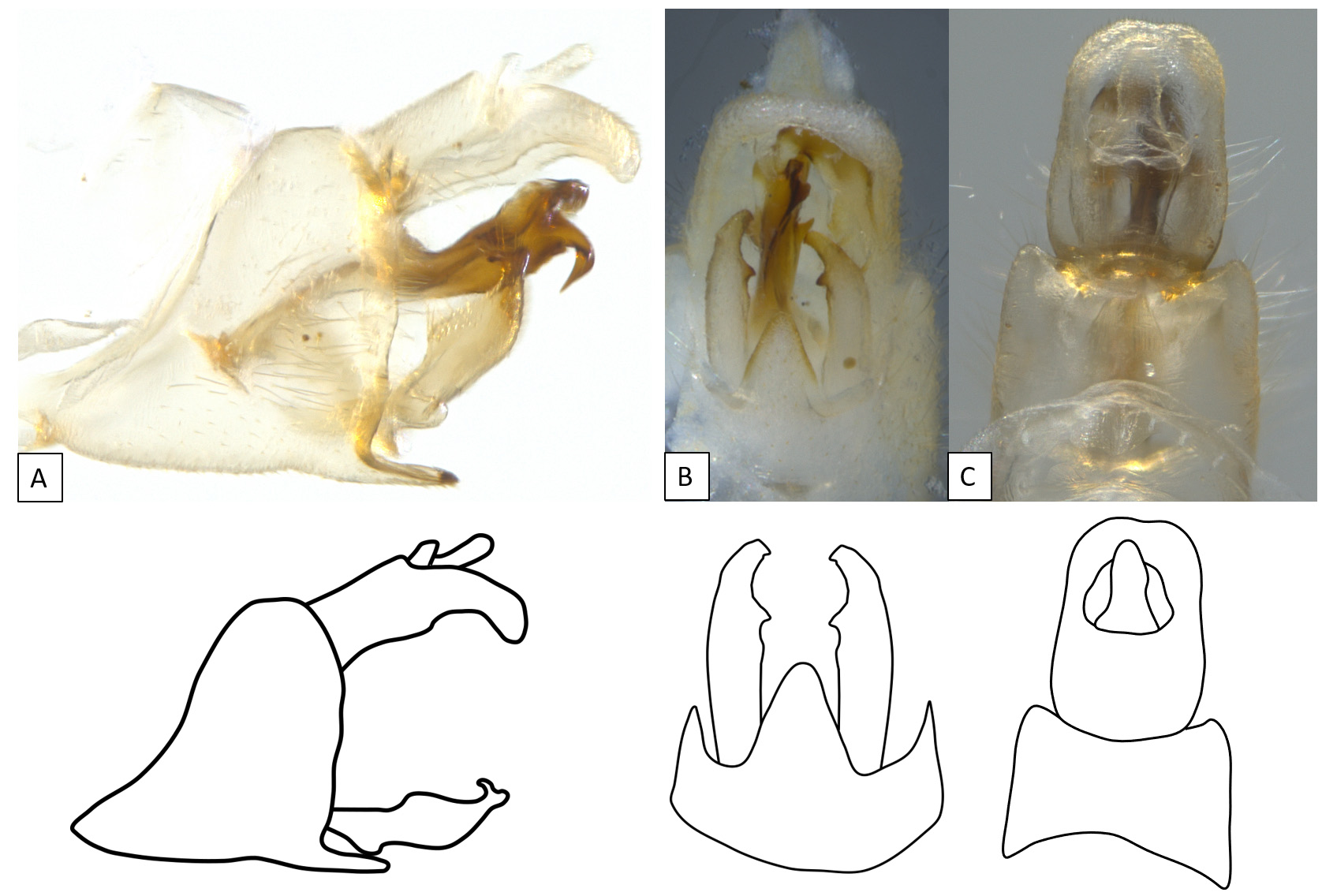

FIGURE 4. Figure 4. Male terminalia of Myxia hernandezi sp. n.; (A) lateral view, (B), ventral view, and (C) dorsal view. 


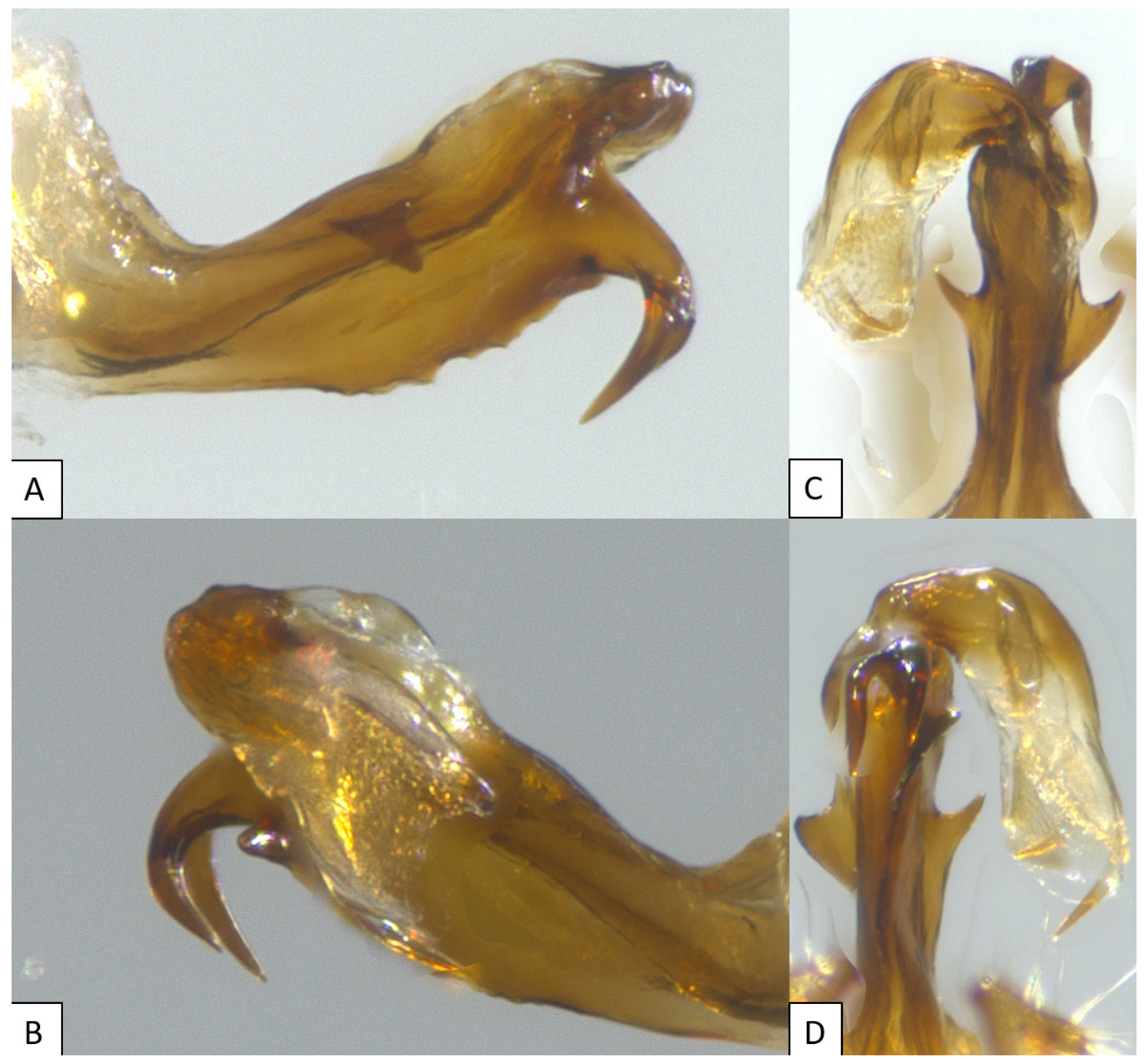

FIGURE 5. Aedeagus and phallobase of Myxia hernandezi sp. n.: (A) left lateral view, (B), right lateral view, (C) dorsal view, and (D) ventral view.

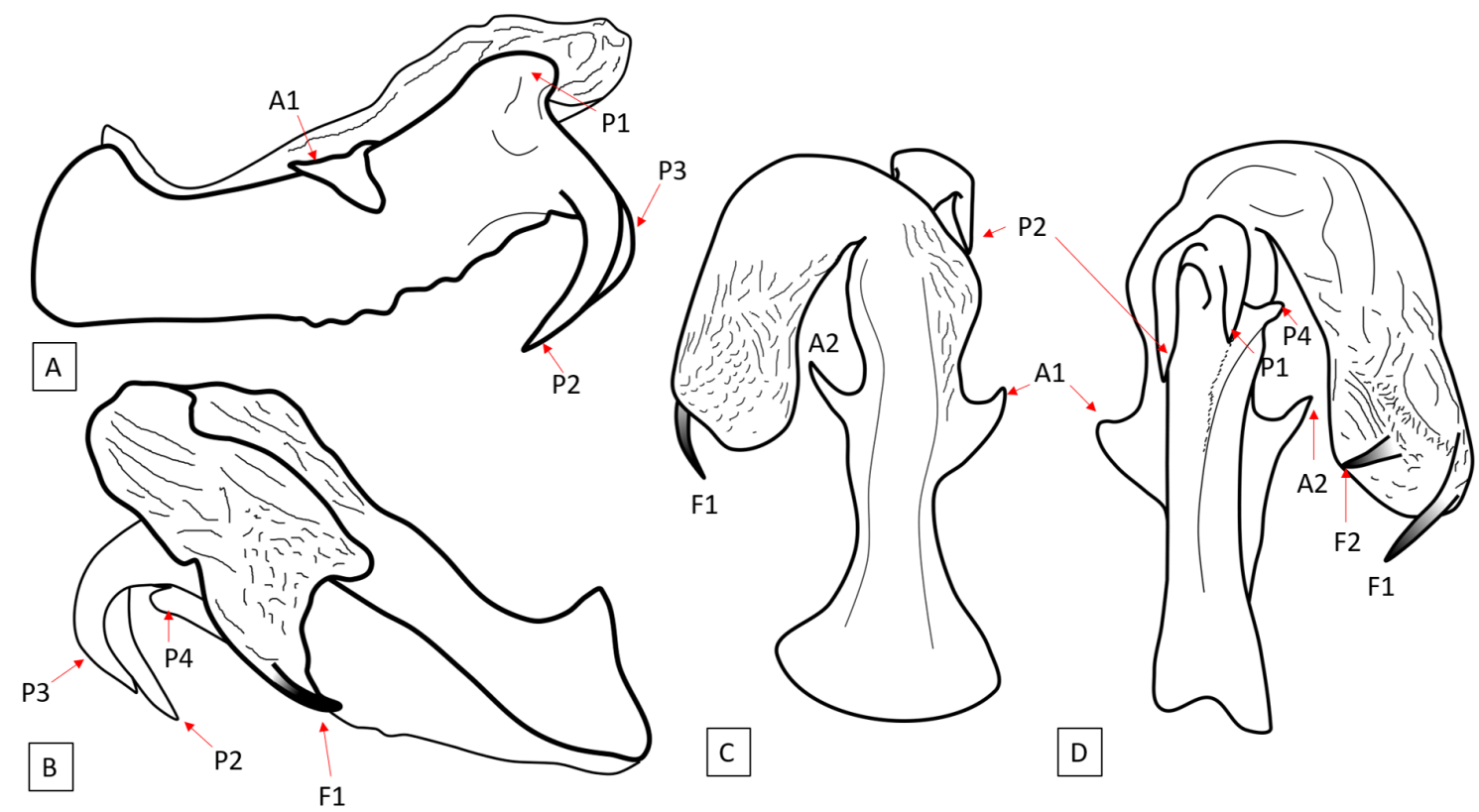

FIGURE 6. Line art of aedeagus and phallobase of Myxia hernandezi sp. n.: (A) left lateral view, (B), right lateral view, (C) dorsal view, and (D) ventral view. 
Etymology. The specific epithet is an honorific for Esteban Josué Hernández Arce at Hotel Villa Blanca, who has provided valuable guidance and information on the habitat where the new species was found and has always supported and been accommodating to research needs. Pura vida primo.

Material examined. Holotype male "Costa Rica, Alajuela / Los Angeles Cloud Forest / Coll.: B.W.Bahder / 16.V.2018 / Host: Geonoma sp. palm // Holotype / Myxia hernandezi" (FSCA) Paratypes, Los Angeles Cloud Forest [16 May 2018] (3 males, FLREC).

Sequence data. For the COI gene, a 543 bp product was generated (GenBank Accession No. MZ234085). Based on the Maximum Likelihood analysis, there was strong bootstrap support (95) for placement of Myxia hernandezi sp. n. adjacent to M. delta within the Myxia clade (Fig. 7). Based on the multiple pairwise comparison, Myxia hernandezi sp. n. differed by an average of 16.5\% ( \pm 2.3$)$ from other species in Myxia with species in Myxia differing by an average of $18.1 \%( \pm 1.4)$ among each other (Table 4). Myxia hernandezi $\mathbf{s p .} \mathbf{n}$. differs by an average of $18.8 \%$ ( \pm 0.3$)$ to members of the genus Haplaxius, $20.4 \%$ to Nymphomyndus caribbea, and $19.2 \%$ to Oecleus mackaspringi (Table 4).

TABLE 4. Pairwise comparison for the COI gene based on 1,000 bootstrap replications using the p-distance method; numbers on bottom left=percent difference, numbers in upper right=standard error.

\begin{tabular}{llllllllllll}
\hline & 1 & 2 & 3 & 4 & 5 & 6 & 7 & 8 & 9 & 10 \\
\hline 1 & Myxia delta & & 0.0164 & 0.0162 & 0.0135 & 0.0168 & 0.0157 & 0.0164 & 0.0162 & 0.0162 & 0.0168 \\
2 & Myxia belinda & 0.1934 & & 0.0168 & 0.0168 & 0.0164 & 0.0156 & 0.0173 & 0.0164 & 0.0171 & 0.0165 \\
3 & Myxia baynardi & 0.1823 & 0.2210 & & 0.0167 & 0.0165 & 0.0158 & 0.0167 & 0.0168 & 0.0170 & 0.0167 \\
4 & Myxia hernandezi sp. n. & 0.1197 & 0.1860 & 0.1860 & & 0.0177 & 0.0171 & 0.0182 & 0.0166 & 0.0169 & 0.0170 \\
5 & Haplaxius dougwalshi & 0.1971 & 0.1989 & 0.1915 & 0.1989 & & 0.0154 & 0.0150 & 0.0136 & 0.0161 & 0.0165 \\
6 & Haplaxius skarphion & 0.1823 & 0.1860 & 0.2063 & 0.2026 & 0.1547 & & 0.0153 & 0.0126 & 0.0154 & 0.0160 \\
7 & Haplaxius pictifrons & 0.1842 & 0.2155 & 0.1989 & 0.2265 & 0.1547 & 0.1565 & & 0.0142 & 0.0159 & 0.0171 \\
8 & Haplaxius crudus & 0.1897 & 0.2026 & 0.1952 & 0.1897 & 0.1197 & 0.1123 & 0.1400 & & 0.0162 & 0.0159 \\
9 & Nymphomyndus caribbea & 0.2044 & 0.2136 & 0.2118 & 0.2044 & 0.1823 & 0.1676 & 0.1750 & 0.1713 & & 0.0158 \\
10 & Oecleus mackaspringi & 0.1915 & 0.1989 & 0.1750 & 0.1768 & 0.1639 & 0.1786 & 0.1989 & 0.1768 & 0.1897 & \\
\hline
\end{tabular}

For the 18S gene, a 1,294 bp product was generated (GenBank Accession No. MZ262449). Based on the Maximum Likelihood analysis, there was strong bootstrap (99) support for the placement of Myxia hernandezi sp. n. in the genus Myxia adjacent to M. delta (Fig. 7). Additionally, there is strong bootstrap support (99) for the genus Myxia overall based on the 18S gene (Fig. 7). Based on the multiple pairwise comparison, Myxia hernandezi sp. n. differed by an average of 1.2\% ( \pm 0.2$)$ from other species in Myxia with species in Myxia differing by an average of $0.9 \%( \pm 0.2)$ among each other (Table 5). Myxia hernandezi sp. $\mathbf{n}$. differs by an average of $2.6 \%( \pm 0.2)$ to members of the genus Haplaxius, 2.8\% to Nymphomyndus caribbea, and 2.6\% to Oecleus mackaspringi (Table 5).

For the H3 gene, a 328 bp product was generated for Myxia hernandezi sp. n. (GenBank Accesion No. MZ274043) as well as all other taxa used for molecular comparisons (Table 2). The Maximum Likelihood phylogeny showed strong bootstrap support (98) for the placement of Myxia hernandezi sp. n. adjacent to M. delta and moderate bootstrap support for Myxia as a monophyletic clade (75) (Fig. 7). The consensus tree generated using COI, 18S, and H3 demonstrated very strong bootstrap support (100) for the placement of Myxia hernandezi sp. n. within the genus Myxia and also demonstrated strong support (96) for Myxia as a clade (Fig. 7). Based on the multiple pairwise comparison, Myxia hernandezi sp. n. differed by an average of $4.6 \%( \pm 1.2)$ from other species in Myxia with species in Myxia differing by an average of $4.9 \%( \pm 0.6)$ among each other (Table 6). Myxia hernandezi sp. n. differs by an average of $12.7 \%( \pm 0.6)$ to members of the genus Haplaxius, $10.4 \%$ to Nymphomyndus caribbea, and $12.2 \%$ to Oecleus mackaspringi (Table 6 ). 
TABLE 5. Pairwise comparison for the $18 \mathrm{~S}$ gene based on 1,000 bootstrap replications using the p-distance method; numbers on bottom left=percent difference, numbers in upper right $=$ standard error.

\begin{tabular}{llllllllllll}
\hline & 1 & 2 & 3 & 4 & 5 & 6 & 7 & 8 & 9 & 10 \\
\hline 1 & Myxia baynardi & & 0.0031 & 0.0031 & 0.0027 & 0.0043 & 0.0048 & 0.0045 & 0.0041 & 0.0047 & 0.0046 \\
2 & Myxia hernandezi sp. $\mathbf{n}$. & 0.0141 & & 0.0007 & 0.0025 & 0.0040 & 0.0043 & 0.0038 & 0.0038 & 0.0043 & 0.0040 \\
3 & Myxia delta & 0.0141 & 0.0008 & & 0.0025 & 0.0040 & 0.0042 & 0.0038 & 0.0038 & 0.0043 & 0.0039 \\
4 & Myxia belinda & 0.0086 & 0.0086 & 0.0086 & & 0.0037 & 0.0043 & 0.0039 & 0.0035 & 0.0041 & 0.0041 \\
5 & Haplaxius pictifrons & 0.0243 & 0.0211 & 0.0211 & 0.0204 & & 0.0031 & 0.0026 & 0.0018 & 0.0035 & 0.0032 \\
6 & Haplaxius skarphion & 0.0305 & 0.0258 & 0.0251 & 0.0266 & 0.0117 & & 0.0022 & 0.0026 & 0.0044 & 0.0036 \\
7 & Haplaxius dougwalshi & 0.0258 & 0.0211 & 0.0204 & 0.0219 & 0.0086 & 0.0063 & & 0.0020 & 0.0039 & 0.0036 \\
8 & Haplaxius crudus & 0.0227 & 0.0196 & 0.0188 & 0.0188 & 0.0047 & 0.0086 & 0.0055 & & 0.0035 & 0.0031 \\
9 & Oecleus mackaspringi & 0.0282 & 0.0251 & 0.0243 & 0.0243 & 0.0196 & 0.0266 & 0.0219 & 0.0196 & & 0.0038 \\
10 & Nymphomyndus caribbea & 0.0258 & 0.0227 & 0.0219 & 0.0219 & 0.0141 & 0.0180 & 0.0164 & 0.0125 & 0.0219 \\
\hline
\end{tabular}

TABLE 6. Pairwise comparison for the $\mathrm{H} 3$ gene based on 1,000 bootstrap replications using the p-distance method; numbers on bottom left=percent difference, numbers in upper right=standard error.

\begin{tabular}{llllllllllll}
\hline & 1 & 2 & 3 & 4 & 5 & 6 & 7 & 8 & 9 & 10 \\
\hline 1 & Myxia baynardi & & 0.0122 & 0.0123 & 0.0112 & 0.0171 & 0.0173 & 0.0176 & 0.0181 & 0.0171 & 0.0178 \\
2 & Myxia belinda & 0.0579 & & 0.0120 & 0.0120 & 0.0185 & 0.0181 & 0.0183 & 0.0197 & 0.0160 & 0.0180 \\
3 & Myxia hernandezi sp. n. & 0.0579 & 0.0579 & & 0.0076 & 0.0180 & 0.0175 & 0.0185 & 0.0194 & 0.0162 & 0.0183 \\
4 & Myxia delta & 0.0457 & 0.0549 & 0.0213 & & 0.0173 & 0.0173 & 0.0176 & 0.0186 & 0.0165 & 0.0178 \\
5 & Haplaxius pictifrons & 0.1037 & 0.1250 & 0.1159 & 0.1067 & & 0.0141 & 0.0145 & 0.0177 & 0.0206 & 0.0205 \\
6 & Haplaxius crudus & 0.1128 & 0.1250 & 0.1189 & 0.1128 & 0.0701 & & 0.0128 & 0.0175 & 0.0204 & 0.0203 \\
7 & Haplaxius skarphion & 0.1189 & 0.1250 & 0.1280 & 0.1159 & 0.0823 & 0.0610 & & 0.0166 & 0.0211 & 0.0208 \\
8 & Haplaxius dougwalshi & 0.1250 & 0.1463 & 0.1433 & 0.1311 & 0.1128 & 0.1159 & 0.1067 & & 0.0197 & 0.0214 \\
9 & Nymphomyndus caribbea & 0.1037 & 0.1006 & 0.1006 & 0.1037 & 0.1616 & 0.1616 & 0.1646 & 0.1463 & & 0.0182 \\
10 & Oelceus mackaspringi & 0.1220 & 0.1250 & 0.1341 & 0.1250 & 0.1616 & 0.1585 & 0.1616 & 0.1799 & 0.1311 \\
\hline
\end{tabular}

Remarks. The morphological features of Myxia hernandezi sp. n. strongly support its placement within Myxia, particularly the form of the parameres with a dorsal process and the subtended periandrium. Furthermore, there is strong molecular support from three different loci (mitochondrial, nuclear and ribosomal) for the placement of $M y x$ ia hernandezi sp. n. in Myxia. Based on superficial morphological characters and details of the terminalia, Myxia hernandezi sp. n. most closely resembles $M$. delta relative to other described species of Myxia. These similarities also appear to be supported by the molecular evidence available.

\section{Discussion}

Myxia hernandezi sp. n. represents the $4^{\text {th }}$ described species of Myxia, all of which are reported within Mesoamerica, with all four species documented on palms from Costa Rica (Bahder et al. 2019a, 2020; Echavarria et al. 2021). From both available specimens and the citizen science database iNaturalist (viz. https://www.inaturalist. org/observations?place_id=any\&subview=grid\&taxon_id=1075953\&user_id=gernotkunz), there appear to be additional, undescribed taxa of (or near) Myxia from Costa Rica. While the genus Haplaxius is the New World oecleine most commonly connected with palms because of $H$. crudus serving as a vector for palm-infecting phytoplasmas in the Caribbean, there are more species of Haplaxius known from non-palm plant associations ( 21 species, by our count) than those documented from palms ( 5 species). Thus far, Myxia appears to be strictly associated with palms, but that association may not continue to hold upon further survey efforts for these undescribed taxa. The association is fascinating though because Myxia is within the same tribe as Haplaxius and the close association with palms may lead to the discovery of new phytoplasma vectors in the future. 


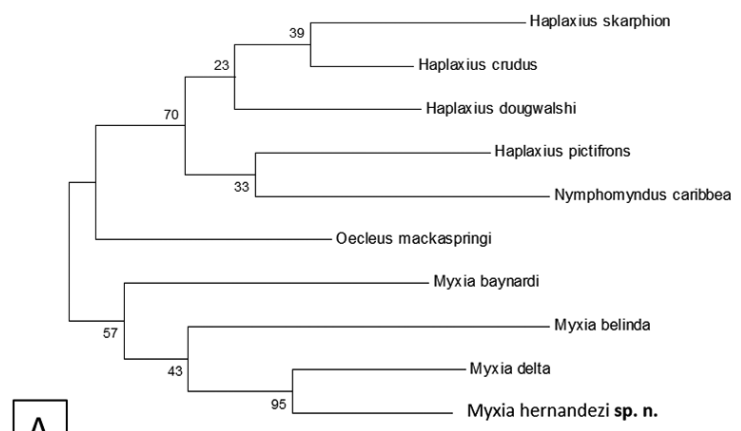

A

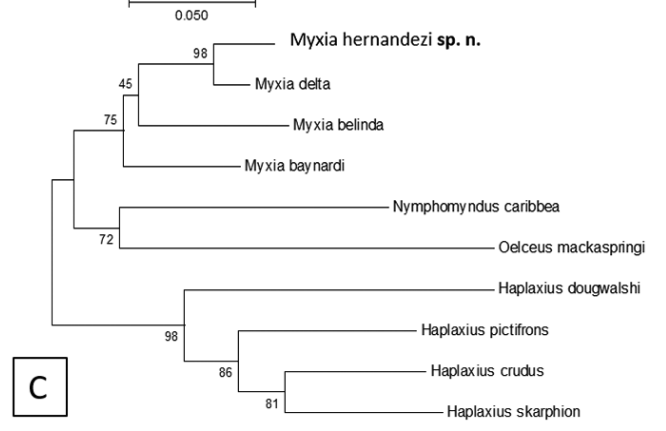

$\widehat{0.0100}$

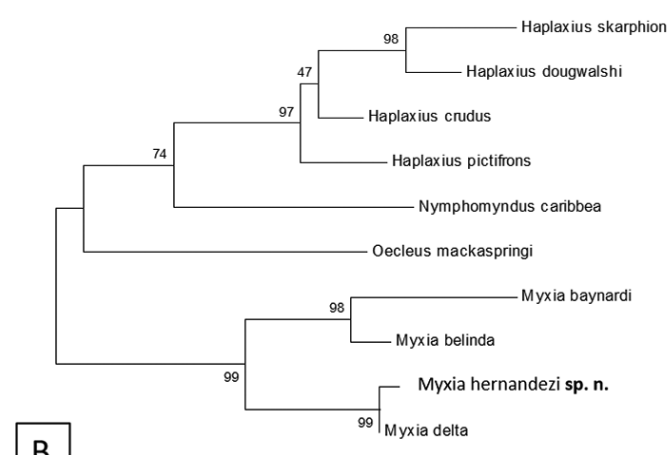

B
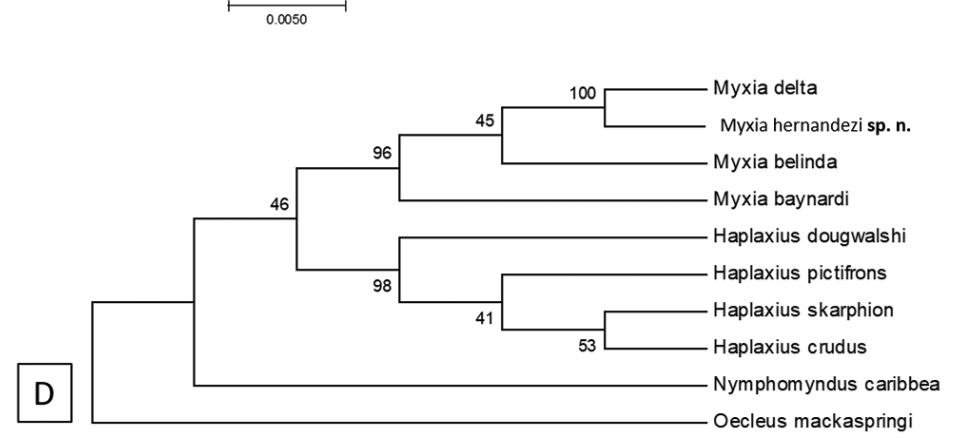

FIGURE 7. Maximum likelihood phylogenetic trees (1,000 replicates) giving phylogenetic position of Myxia hernandezi sp. n. relative to related taxa in Oecleini based on the; (A) COI gene, (B) 18S gene, (C) H3 gene and (D) a consensus tree based on concatenated COI, $18 \mathrm{~S}$ data, and $\mathrm{H} 3$.

\section{Acknowledgements}

The authors are grateful to staff and management at Hotel Villa Blanca for their ongoing support of this research. The authors are grateful to Luz Bahder for translating the abstract into Spanish. Funding for this research was provided University of Florida - Institute of Food and Agricultural Sciences and Emerging Pathogens Institute for providing seed grant funding to support survey work in Costa Rica.

\section{References}

Bahder, B.W., Bartlett, C.R., Barrantes, E.A.B., Zumbado, M.A.Z., Humphries, A.R., Helmick, E.E. Goss, E.M. \& Ascunce, M.S. (2019a) A new genus and species of cixiid planthopper (Hemiptera: Auchenorrhyncha: Fulgoroidea) from the Reserva Privada el Silencio de Los Angeles Cloud Forest in Costa Rica. Zootaxa, 4701 (1), 65-81. https://doi.org/10.11646/zootaxa.4701.1.5

Bahder, B.W., Bartlett, C.R., Barrantes, E.A.B., Echavarria, M.A.Z., Humphries, A.R., Helmick, E.E., Ascunce, M.S. \& Goss, E.M. (2019b) A new species of Omolicna (Hemiptera: Auchenorrhyncha: Fulgoroidea: Derbidae) from coconut palm in Costa Rica and new country records for Omolicna brunnea and Omolicna triata. Zootaxa, 4577 (3), 501-514. https://doi.org/10.11646/zootaxa.4577.3.5

Bahder, B.W., Barrantes, E.A.B., Echavarria, M.A.Z., Mou, D., Helmick, E.E. \& Bartlett, C.R. (2020) A new species of planthopper in the genus Haplaxius (Hemiptera: Auchenorrhyncha: Fulgoroidea: Cixiidae) on palms in Costa Rica and a new county record for Haplaxius skarphion. Zootaxa, 4767 (4), 543-553. https://doi.org/10.11646/zootaxa.4767.4.4

Ball, E.D. (1902) Some new North American Fulgoridae. The Canadian Entomologist, 34, 147-157. https://doi.org/10.4039/Ent34147-6

Barrantes, E.A.B., Echavarria, M.A.Z., Bartlett, C.R., Helmick, E.E. \& Bahder, B.W. (2021) A new species of planthopper in the genus Haplaxius (Hemiptera: Auchenorrhyncha: Fulgoroidea: Cixiidae) from coconut palm (Cocos nucifera) in Costa Rica. Zootaxa, 4963 (3), 412-422.

https://doi.org/10.11646/zootaxa.4963.3.2 
Bourgoin, T. (1988) A new interpretation of the homologies of the Hemiptera male genitalia illustrated by the Tettigometridae (Hemiptera, Fulgoromorpha). In: Vidano, C. \& Arzone, A. (Eds.), Proceedings of the $6^{\text {th }}$ Auchenorrhyncha Meeting, Turin, Italy, September 7-11, 1987. Consiglio Nazionale delle Ricerche, IPRA, Rome, pp. 113-120.

Bourgoin, T. \& Huang, J. (1990) Morphologie compare des genitalia males des Trypetimorphini et remarques phylogénétiques (Hemiptera: Fulgoromorpha: Tropiduchidae). Annales de la Société entomologique de France, 26, 555-564.

Bourgoin, T., Wang, R.R., Ache, M., Hoch, H., Soulier-Perkins, A., Stroinski, A., Yap, S. \& Szwedo, J. (2015) From micropterism to hyperpterism: recognition strategy and standardized homology-driven terminology of the forewing venation patterns in planthoppers (Hemiptera: Fulgoromorpha). Zoomorphology, 134 (1), 63-77. https://doi.org/10.1007/s00435-014-0243-6

Dzido, J.L., Sánchez, R., Dollet, M., Julia, J.F., Narvaez, M., Fabre, S. \& Oropeza, C. (2020) Haplaxius crudus (Hemiptera: Cixiidae) transmits the lethal yellowing phytoplasmas, 16SrIV, to Pritchardia pacifica Seem. \& H. Wendl (Arecaceae) in Yucatan, Mexico. Neotropical Entomology, 49 (6), 795-805. https://doi.org/10.1007/s13744-020-00799-2

Echavarria, M.A.Z., Barrantes, E.A.B., Bartlett, C.R., Helmick. E.E. \& Bahder, B.W. (2021) A new species of planthopper in the genus Myxia (Hemiptera: Auchenorrhyncha: Cixiidae) from the Reserva Privada el Silencio de Los Angeles Cloud Forest in Costa Rica. Zootaxa, 4915 (3), 351-363. https://doi.org/10.11646/zootaxa.4915.3.4

Emeljanov, A.F. (1989) On the problem of division of the family Cixiidae (Homoptera, Cicadina). Entomological Review, 68 (4), 54-67.

Emeljanov, A.F. (1996) On the system and phylogeny of the family Derbidae (Homoptera, Cicadina). Entomological Review, 75 (2), 70-100.

Fennah, R.G. (1971) Fulgoroidea from the Cayman Islands and adjacent Areas. Journal of Natural History, 5, $299-342$. https://doi.org/10.1080/00222937100770241

Fowler, W.W. (1904) Order Rhynchota. Suborder Hemiptera-Homoptera. (Continued). Biologia Centrali-Americana; contributions to the knowledge of the fauna and flora of Mexico and Central America, 1, 85-124.

Harrison, N.A., Helmick, E.E. \& Elliott, M.L. (2008) Lethal-yellowing type diseases of palms associated with phytoplasmas newly identified in Florida, USA. Annals of Applied Biology, 153 (1), 85-94. https://doi.org/10.1111/j.1744-7348.2008.00240.x

Howard, F.W. \& Thomas, D.L. (1980) Transmission of palm lethal decline to Veitchia merrillii by a planthopper Myndus crudus. Journal of Economic Entomology, 73 (5), 715-717. https://doi.org/10.1093/jee/73.5.715

Folmer, O., Black, M., Hoeh, W., Lutz, R. \& Vrijenhoek, R. (1994) DNA primers for amplification of mitochondrial cytochrome $c$ oxidase subunit I from diverse metazoan invertebrates. Molecular Marine Biology and Biotechnology, 3 (5), $294-299$.

Kramer, J.P. (1979) Taxonomic study of the planthopper genus Myndus in the Americas (Homoptera: Fulgoroidea: Cixiidae). Transactions of the American Entomological Society, 105 (3), 301-389.

Kumar, S., Stecher, G. \& Tamura, K. (2016) MEGA7: Molecular Evolutionary Genetics Analysis version 7.0 for bigger datasets. Molecular Biology and Evolution, 33, 1870-1874.

https://doi.org/10.1093/molbev/msw054

Muir, F.A.G. (1922) New Malayan Cixiidae (Homoptera). Philippine Journal of Science, 20, 111-119.

Myrie, W., Helmick, E.E., Bartlett, C.R., Bertacini, A. \& Bahder, B.W. (2019) A new species of planthopper belonging to the genus Oecleus Stål, 1862 (Hemiptera: Fulgoroidea: Cixiidae) from coconut palm (Cocos nucifera L) in Jamaica. Zootaxa, 4712 (1), 127-137. https://doi.org/10.11646/zootaxa.4712.1.9

Simon, C., Frati, F., Beckenbach, A., Crespi, B., Liu, H. \& Flook, P. (1994) Evolution, weighting, and phylogenetic utility of mitochondrial gene sequences and a compilation of conserved polymerase chain reaction primers. Annals of the Entomological Society of America, 87 (6), 651-701. https://doi.org/10.1093/aesa/87.6.651

Spinola, M. (1839) Essai sur les Fulgorelles, sous-tribu de la tribu des Cicadaires, ordre des Rhyngotes. Annales de la Société Entomologique de France, 8, 133-337.

Stål, C. (1862) Novae vel minus cognitae Homopterorum formae et species. Berliner Entomologische Zeitschrift, 6, 303-315. https://doi.org/10.1002/mmnd.47918620303

Van Duzee, E.P. (1907) Notes on Jamaican Hemiptera: A report on a collection of Hemiptera made on the island of Jamaica in the spring of 1906. Bulletin of the Buffalo Society of Natural Sciences, 8 (5), 3-79. 